\title{
An Image Processor Bill Acceptor for Iraqi Currency
}

\author{
Ammar Awni Abbas \\ Computer Center, University of Baghdad, Baghdad-Iraq. \\ Corresponding Author:Ammarawni@uob.edu.iq.
}

\begin{abstract}
Automatic Teller Machines (ATMs) and vending machines is a rapidly growing trade in the Iraqi market. Bill acceptors lvalidators, are considered the heart of these machines have a great significance. Due to the inflation in Iraq with numerous forgery attempt to the currency, an efficient way to accept and validate the Iraqi currency is very important. This paper suggests a bill acceptorlvalidator to accept, recognize the amount, and validate the bill based solely on image processing functions rather than the magnetic field detection that is used in the regular Bill Acceptors. The program couples the faked bill with a photo graph of the person who presents it. The program uses the Matlab computer vision abilities to recognize the bill. The distinctive characteristics that appears on the banknote when an ultraviolet light is shed on the bill it used for the authentication part with the same Matlab pattern recognition functions. The proposed system include all the mechanical and electronical parts that are used in the proposed system. The results was $100 \%$ accurate, which implies that there is great possibility to use this technique in industrial bill acceptors.

[DOI: 10.22401/ANJS.22.2.10]
\end{abstract}

Keywords: Bill Acceptors, Validators, Matlab, currency authentication, Image Processing.

\section{Introduction}

The Iraqi currency system has been renewed completely after the 2003 events, new bills with a very high security standards was pumped into the circulation in an unprecedented way. Despite the high security measures that has been taken to prevent forgery, the Iraqi currency was subjected to numerous forgery attacks specially the high amount bills. This results in great disturbance in the markets and consequently in the society in general. While the brightness and the texture characteristics of the note may fade away with time, other characteristics like Identification mark, security thread, latent image and watermarks should be persistent with time and not affected with degradation [4]. This paper targets an authentication feature in the Iraqi currency that is ultraviolet illumination. An integrated ultraviolet fluorescent fiber which creates a very special shapes and colors when the note is exposed to an ultraviolet source of light. This property is used in this paper as the main authentication characteristics. The second section of the paper reviews the work of other authors, the third section outlines the proposed system (both hardware and software), and the fourth section discusses the way the apparatus process each bill, the fifth section lists the results for the operation, the sixth section discusses results and main possible enhancement for the suggested apparatus; the seventh section is the references.

\section{Related Work}

A portable X-ray fluorescence (pXRF) spectrometer is used to detect the Peruvian banknotes by comparing the spectra of original and fake notes, Peruvian Nuevo Sol banknotes were studied: 4 genuine and 3 fake ones, in 11 points of analysis for each one, with 77 data set. The correlation analysis of spectra among original notes was 1.0 , and there was no correlation with fake banknotes [1]. The most frequent related work is dedicated to currency recognition using image processing and the visual characteristics of the note as [2]. A combined method between Principal Component Analysis (PCA) and back propagation neural network (BPNN) is proposed by [3] to detect banknotes, for the 24 contrast experiments, the training set composed of the banknote images captured by webcam results in better recognition results than the training set made up of scanned banknote images, the highest average F-measure is 0.9980 obtained by combination No.9 (Color+LBP_PCA_BPNN) when using the banknote images captured by webcam for 
training. In [4] a complete survey for all fields of currency detection and authentication plus recognition of the serial number of the banknote is presented, Techniques used in each of the four areas recognize banknote information (denomination, serial number, authenticity, and physical condition) based on image or sensor data, and are actually applied to banknote processing machines. A scaleinvariant feature transform (SIFT) algorithm combined with mobile camera is used to recognize the currency [5], the correct recognition average for the color SIFT is 0.79 While, for Gray SIFT the correct recognition average is 0.53 . Similarity mapping used to recognize banknotes images captured by light sensor in [6], the proposed method has an error rate of 0.002 with rejection rate of 0.004 which is much better than other methods. In paper [7], the authors tried to use simple algorithms to increase the speed of recognition for the Chinese yuan (RBM) with great efficiency, the recognition ratio of the $(100,50$ old style, 50 new style, 20 and 10 of the RBM bill) is (95\%, $99 \%, 99 \%, 92 \%$ and $98 \%)$ respectively. In [8] an image processing technique is used for the recognition process in Matlab program, the authentication of the 100 teka (currency of Bangladesh) is made with $100 \%$ success rate. [9] Proposed a radial basis for classification that uses basic features with correlation to detect the currency, the overall Average Recognition Rate for the data of 110 images is computed as $91.51 \%$. The proposed algorithm is fully automatic and requires no human intervention. The characters of the note is heuristically analyzed to get the exact features of them before the recognition in [10], Heuristic analysis of Serial Number for 9 character, taking in account four individual properties of a character(hue, saturation, contrast and brightness) reveals that particular trend in terms of values of hue and saturation, contrast and brightness. It can be concluded that while contrast and brightness are dependent on the extrinsic factors of sensor, hue and saturation are more likely dependent on the consistent pattern design of note. In [11] a review of the recognition processes is made. After applying certain filters to classify the acquired image a neural networks method for identification is used by [12], making a universal recognition program for all currencies is the main objective for that paper, the proposed algorithm was tested on five currencies with huge success. The process of recognition using Matlab with the most common security characteristics is discussed in [13]. An embedded system for detecting the fake currency is proposed by [14], which relied on some special currency characteristics, a neural network is used for recognition and a Light Emitting Diode (LED) for authentication. The success rate was $100 \%$. In [15] the shape and color information is used in the algorithm, two currencies are used in the data base of the program. The results showed that the scanned images have better results than images taken by a digital camera. In [16] an ensemble neural network (ENN) is used, by using negative correlation learning (NCL) the system gains experience on different parts or portion of input patterns in an ensemble. After applying certain filters to classify the acquired image a neural networks method for identification is used by [17]. [18] Used the mobile phone camera to make the recognition in real time to help people with impaired visual conditions.

\section{Instrument and software setting}

The proposed system is shown in Fig.(1), it's composed of the following Hardware parts:

1- Motion sensor, to detect the currency is being presented by the user.

2- Servo motor to roll the paper in, when it gets the signal from the motion sensor.

3- Plastic clear Chamber, which consists of two layers of plastic plates joined together by glue forming a clear chamber to host the rolled in note, the servo motor (1) roll in the banknote and let it lay on this chamber for further processing.

4- Web camera (1) for the recognition and authentication, this camera faces the plastic chamber from horizontal view.

5- Arduino Uno microcontroller to connect to the relay.

6- Two-Channel relay: connected to the Arduino Uno which is used to activate/deactivate the White and Ultraviolet lights.

7- White bulb light: used for recognition process. 
8- Ultraviolet light: used for the authentication process.

9- Servo motor (2): used to roll the paper out of the plastic chamber when the recognition/authentication processes is over, the note is guided to the currency accumulation sack.

10- Web camera (2): to take a photograph for the Fake currency provider.

All of these components are encapsulated in well isolated box to prevent the effects of any unwanted lights. As shown in Fig.(1).

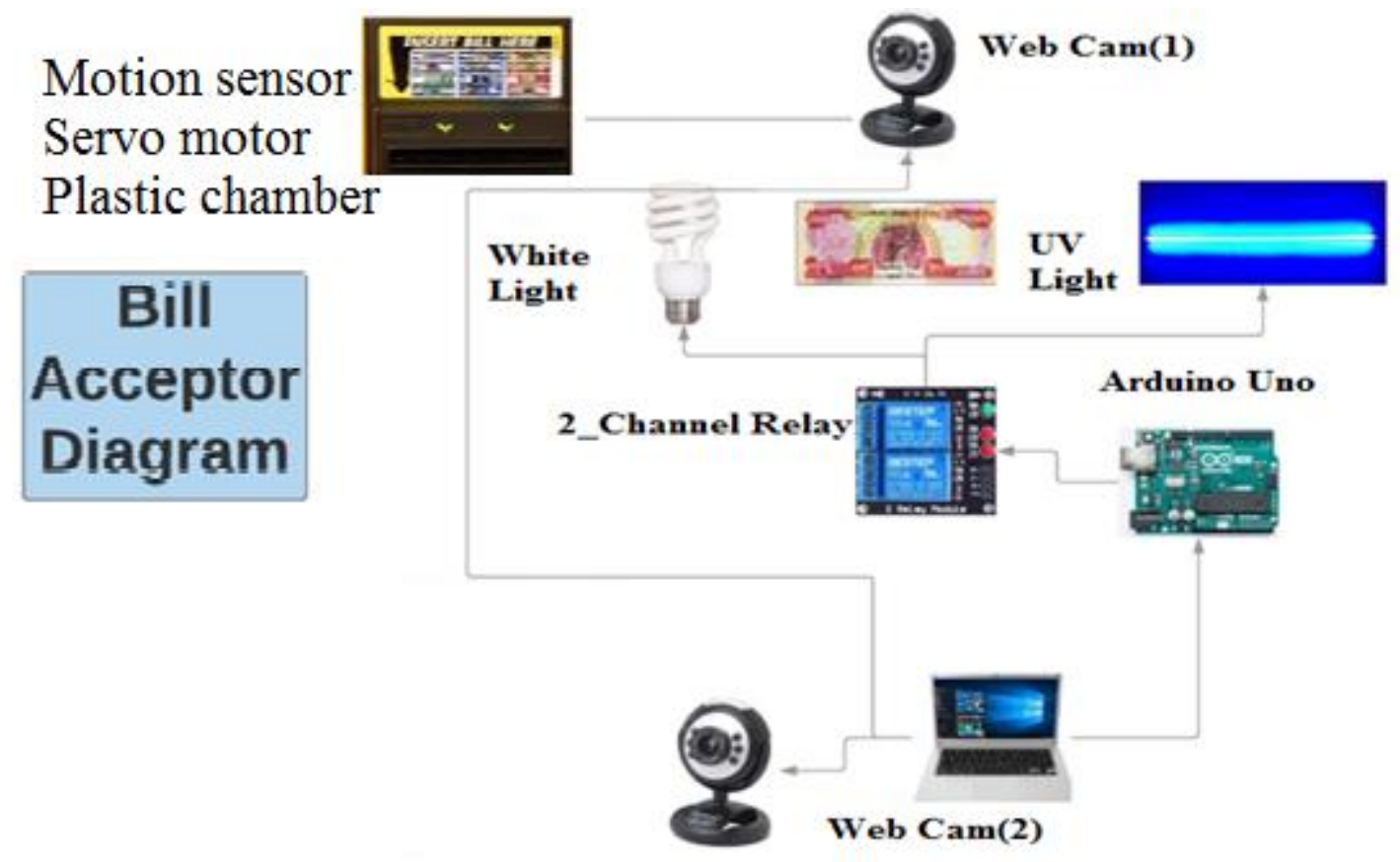

Fig.(1): The proposed Bill Acceptor Hardware.

The apparatus is programmed using Matlab programming language, with the following additional packages installed:

1. Matlab Support Package for USB Web cams.

2. Matlab Support Package for Arduino Hardware (Arduino microcontroller can be replaced by the Raspberry Pi microcontroller).

The following Matlab toolboxes is used and must be installed:

1. Computer Vision System Toolbox.

2. Image Acquisition Toolbox.

3. Image Processing Toolbox.

A flowchart of the proposed bill acceptor operation is shown in Fig.(2). 


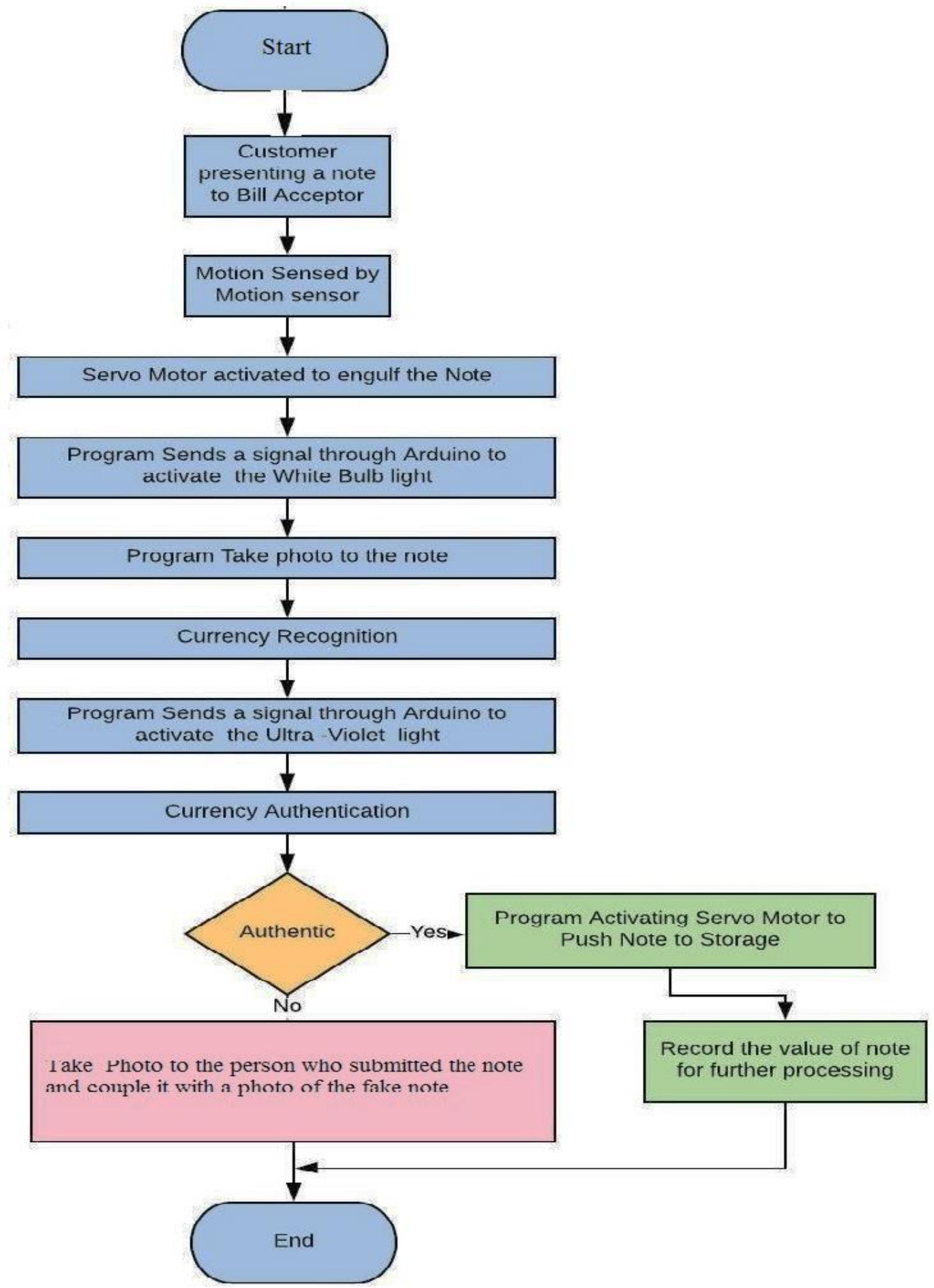

Fig.(2): A Flowchart of the Bill Acceptor operation.

\section{Test}

A bill acceptors have one basic purpose is to accept and validate paper currency in exchange for a product or service. The bill acceptor "grabs" the bill, scans it for validity, and sends a corresponding Input/Output signal to its output connector to record the transaction. The proposed device process the bill as follows:

1- The bill presented to the proposed device is sensed by motion sensor, that in turn, the sensor sends a signal to a servo motor to operate, the motor attached to serrated roller, this event suck the bill inside the machine, another signal is sent to trigger the start of the Matlab program. The note is guided to the clear plastic chamber through a proper plastic guide; this chamber serve as temporary station, where the recognition and the authentication is made. 
2- The system is connected via Arduino Uno microcontroller to a two channel-relay that switch $\mathrm{ON}$ an ordinary white light, followed by, a taking a photo in shape of frame captured from the live feed of web camera (1) attached to the system, the photo is used to recognize the banknote amount by applying the pattern recognition software between the target image and a set of templates that is a scanned image of the various denomination of notes.

3- The program send a signal to the Arduino Uno microcontroller, which in turn sends a signal to the two channel relay to turn ON the Ultraviolet light.

4- Another photo is taken from the web camera (1) is taken when the Ultraviolet light is $\mathrm{ON}$, and the same recognition program is activated with another set of templates that is made with Ultraviolet light. This photo is used to authenticate the currency.

5- If the currency is authentic, a servo motor (2) is turned ON to push the currency to the currency accumulation sack. With the value registered in the system for further operations.

6- Otherwise, the servo motor(2) also turned ON to push the currency to the accumulation sack with a flag raised to program to take a photo of the person who present the fake banknote using web camera (2). This will prevent fake currency from circulating in the monetary system.

The recognition process is made by exploiting the capabilities of the computer vision toolbox in MATLAB. By discovering point correspondences between the reference templates and the objective picture, the matching is made when the template image that has the largest point correspondences with the target image.

The proposed program is based upon the Speeded-Up Robust Features (SURF) which uses scale- and rotation-invariant detector and descriptor. The main idea is to use integral images for image convolutions.

There are eight denominations in the Iraqi currency making a total of sixteen template pictures that the program must choose from to detect the amount of the banknote. The process of currency recognition consists of five steps that is repeated between the target image and each of the templates:

1- Read both the Template images and the target image.

2- Detect feature point of both images using the detectSURFFeatures function, this function returns a Speeded-Up Robust Features (SURF) Points object, points, containing information about SURF features detected in the 2-D grayscale input image I. The detectSURFFeatures function implements the Speeded-Up Robust Features (SURF) algorithm to find blob features.

3- Extract feature descriptors: using extractFeatures function which Extract interest point descriptor. The function derives the descriptors from pixels surrounding an interest point. The pixels represent and match features specified by a single-point location. Each single-point specifies the center location of a neighborhood. The method used for descriptor extraction depends on the class of the input points, in this case we used Speeded-Up Robust Features (SURF) obtained from the second stage.

4- Find the putative point matches: using the matchFeatures function which match the features using their descriptors. This function returns indices of the matching features in the two input feature sets obtained from Templates and target images. The input feature is a binary Features image.

5- The program detects the matched features between the target and each of the template, the image with the largest match points is considered to be a hit.

The same procedure is repeated for the authentication process with the exception that the ultraviolet light is turned $\mathrm{ON}$ when the webcam takes the photo and same applied to the template images. 


\section{Experiments and Results}

The experimental stage consists of recognizing and authenticating a set of 2400 banknotes, 300 banknotes per denomination class. The notes are acquired from the ordinary monetary circulation where the bills are sometimes partially torn or handwritten notes. The results of the recognition part is shown in Figs. [3, 4, 5, 6and 7]. Table (1) shows the results of the testing.

Table (1)

The Recognition and Authentication results.

\begin{tabular}{|c|c|c|c|c|c|c|c|c|}
\hline Banknote & $\begin{array}{c}50 \\
\text { Dinars } \\
\end{array}$ & $\begin{array}{c}250 \\
\text { Dinars }\end{array}$ & $\begin{array}{c}500 \\
\text { Dinars }\end{array}$ & $\begin{array}{c}1000 \\
\text { Dinars }\end{array}$ & $\begin{array}{l}5000 \\
\text { Dinars }\end{array}$ & $\begin{array}{l}\text { 10000 } \\
\text { Dinars }\end{array}$ & $\begin{array}{l}25000 \\
\text { Dinars }\end{array}$ & $\begin{array}{l}50000 \\
\text { Dinars }\end{array}$ \\
\hline $\begin{array}{l}\text { No. of authentic } \\
\text { notes tested }\end{array}$ & 290 & 290 & 290 & 290 & 290 & 290 & 290 & 290 \\
\hline $\begin{array}{l}\text { No. of forged notes } \\
\text { tested }\end{array}$ & 10 & 10 & 10 & 10 & 10 & 10 & 10 & 10 \\
\hline Total & 300 & 300 & 300 & 300 & 300 & 300 & 300 & 300 \\
\hline Recognition & 300 & 300 & 300 & 300 & 300 & 300 & 300 & 300 \\
\hline Authentication & 300 & 300 & 300 & 300 & 300 & 300 & 300 & 300 \\
\hline Error & 0 & 0 & 0 & 0 & 0 & 0 & 0 & 0 \\
\hline Accuracy & $100 \%$ & $100 \%$ & $100 \%$ & $100 \%$ & $100 \%$ & $100 \%$ & $100 \%$ & $100 \%$ \\
\hline
\end{tabular}

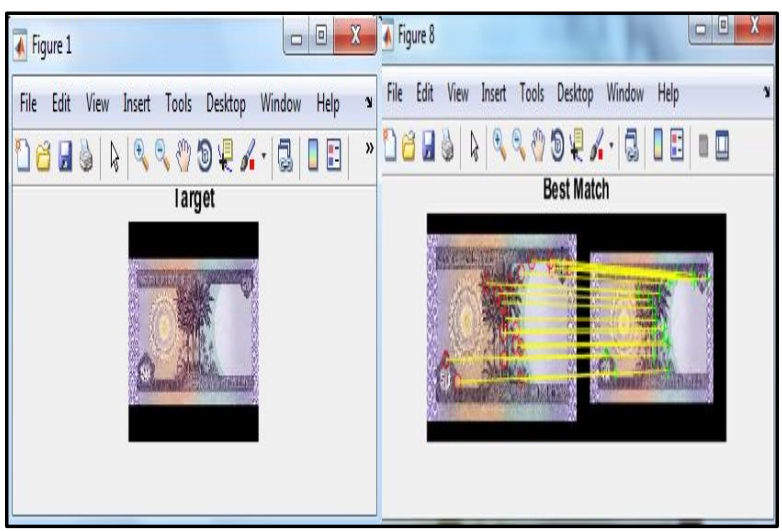

Fig.(3): Matching of 50 Dinar denomination (Back).

In Fig (3) the target image on the left that is processed by the suggested device and the match is found, for descriptive reasons the target and the best match are presented in the same image on the right with the match points on both images illustrated on both images.

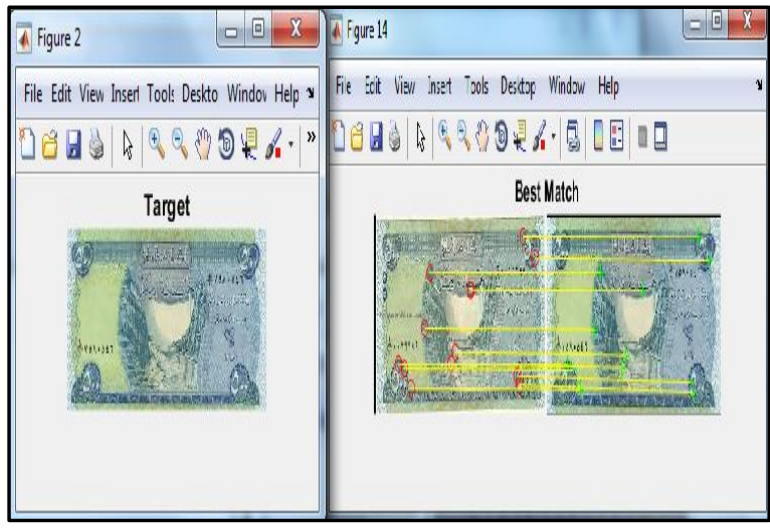

Fig.(4): Matching of 500 Dinar denomination (Front).

The target image on the left and the best match coupled with the target on the right for the 500 dinar denomination. 


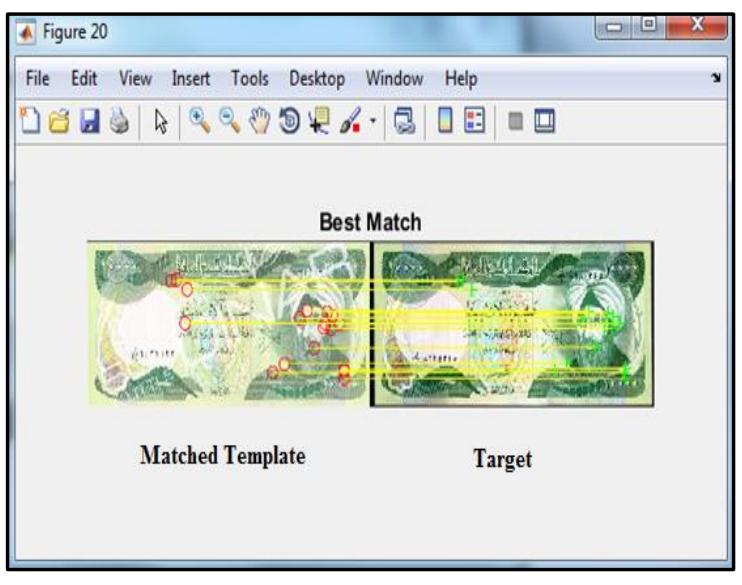

Fig.(5): Matching of 10000 Dinar denomination (Front).

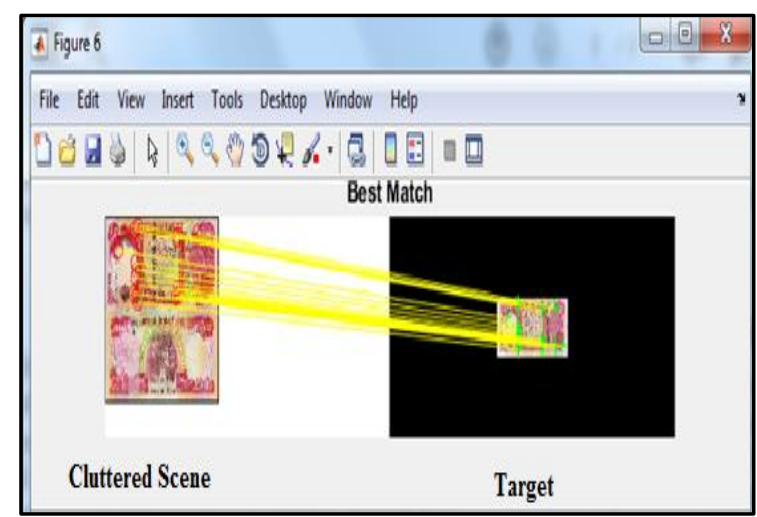

Fig.(6): Matching of 25000 Dinar denomination (In Cluttered Scene).

The target image on the right can be detected even if the best match found in a scene that contains both faces of the currency as shown in Fig.(6).

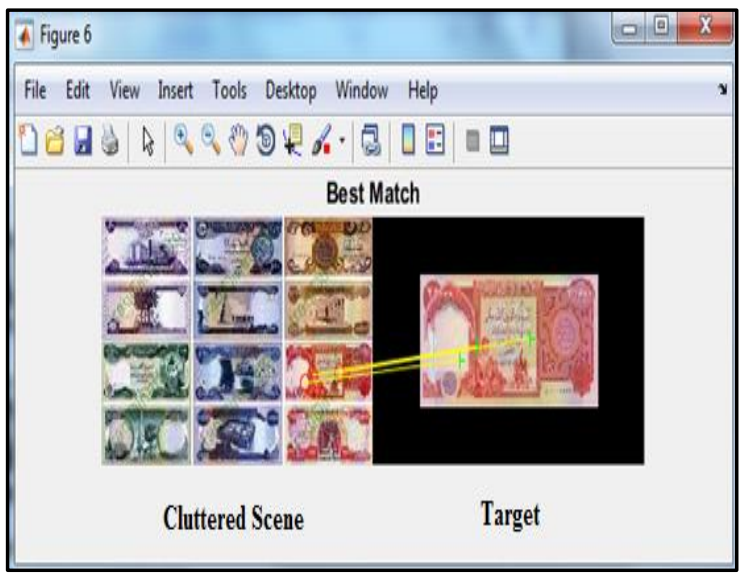

Fig.(7): Matching of 25000 Dinar denomination (In Complex Cluttered Scene).

In Fig.(7) the match for the target image no matter how complex the scene is complicated and no matter the difference in the size of the target and the templates.

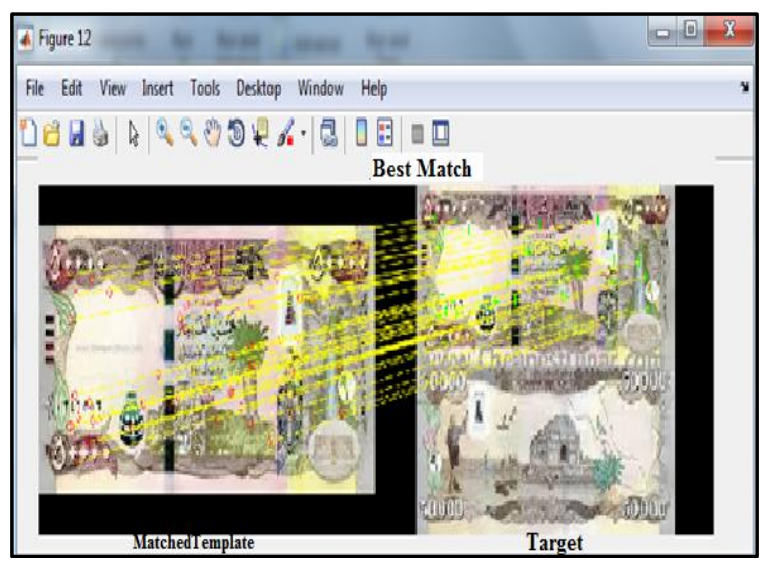

Fig.(8): Matching of 50000 Dinar denomination (2-Sides of the Note).

In Fig.(8) we scanned two side of the bill as one target image and again the program succeed in finding the best match.

The accuracy of the results is $100 \%$, the pattern matching method used is both concise and precise. The clustering of instructions in the form of functions in MATLAB creates the ability to get the maximum functionality with a minimum amount of lines of code. MATLAB support for microcontrollers, extended the capabilities of MATLAB to a whole new level that can be used to create a very wide mechatronics applications including the proposed bill acceptor. Computer vision toolbox made the recognition process easy, fast and accurate. The target image can be identified even if the note is placed in a cluttered scene or if the target is rotated or partially torn. As a result, there is no need for preprocessing, image registration, or any other enhancement methods. For the authentication part only the part that contains the ultraviolet sensitive fiber is used for the pattern matching, this part is cropped from the templates (exposed to ultraviolet light), as shown in Fig.(9).

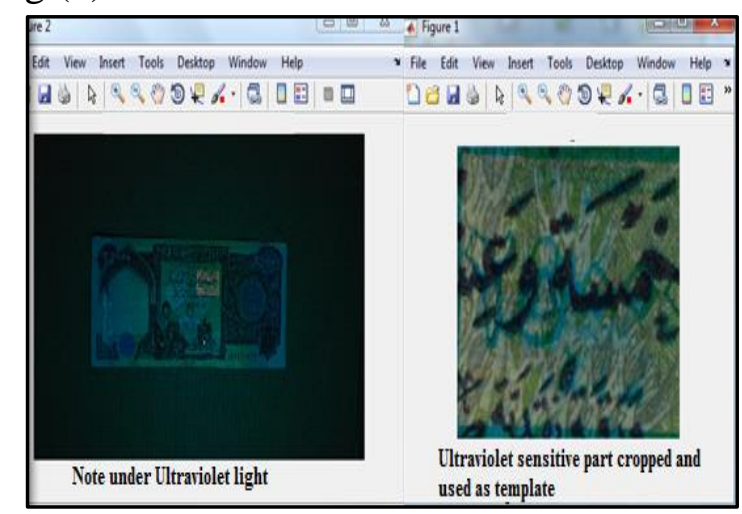

Fig.(9): Cropping the Ultraviolet part of the note. 
The region of interest ROI in case of authentication is the phosphoric rectangle that appears after shedding the ultraviolet light, cropping that part, speeds up the process of authentication and eliminates the use of unnecessary preprocessing operation. The program couples the currency denomination that is used as target for the recognition processing with a corresponding phosphoric rectangle for that certain currency to be used for the authentication process.

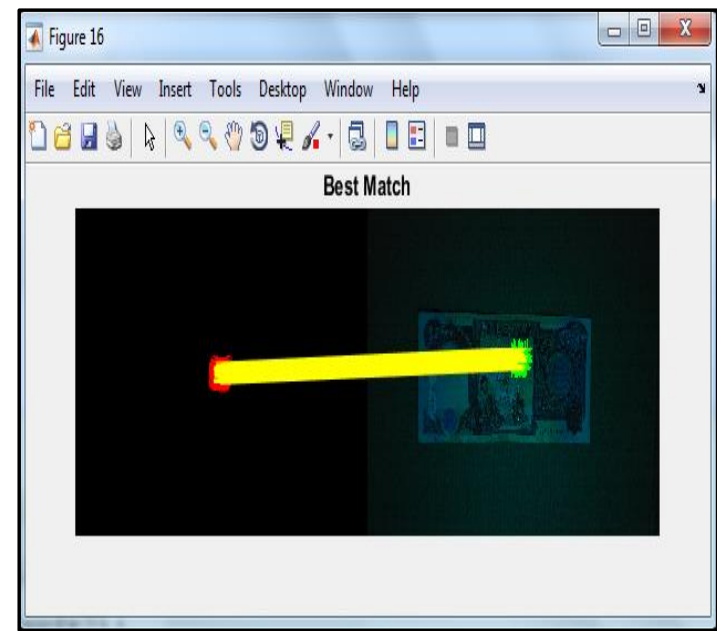

Fig.(10): The Note is authentic, a match found between target and template.

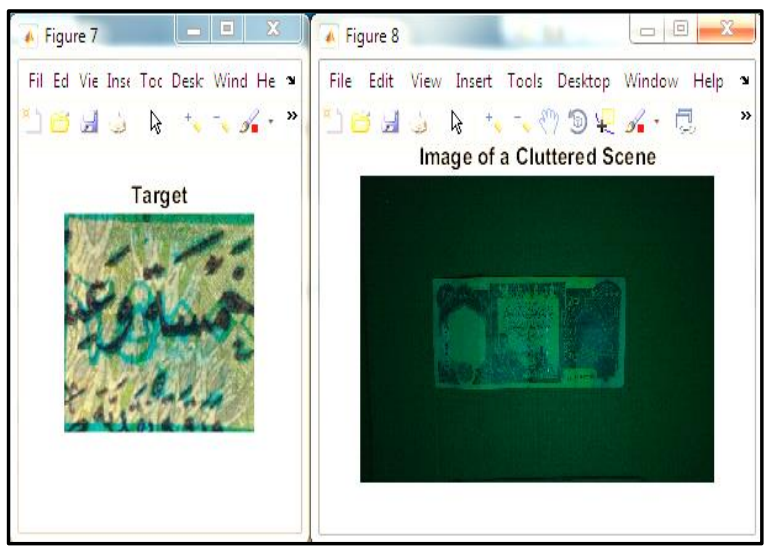

Fig.(11): The Note is forged, No match found between target and template.

The algorithm will find no match for the phosphoric rectangle in the forged note as shown in Fig.(11).

\section{Discussions}

The suggested configuration can be turned into a standalone device. The algorithm can be uploaded to the microcontroller such as Arduino or Raspberry Pi where the webcams can be replaced by (OV 7670for Arduino or
Raspberry Pi Camera Module v2). Both microcontrollers are supported by Matlab and can benefit from the computer vision capabilities of Matlab. The algorithm is very robust, it can distinguish images in spite of a scale change, rotation, background color or impediment. The computer vision functions minimize the amount of coding needed while retaining high speed, so there is a possibility of operation in real time. To further enhance the work by taking the following measures:

1.Adding more security features to be checked by the algorithm such as security thread and water marks.

2.The ability to recognize and authenticate currencies other than the Iraqi currency, by expanding the algorithm to accept other banknotes templates.

\section{References}

[1] Jara M., Obregón C., Del Castillo C. "Exploratory analysis for the identification of false banknotes using portable X-ray Fluorescence spectrometer", Applied Radiation and Isotopes, 135(1), 212-218, 2018.

[2] Rajebhosale S., Gujarathi D., Nikam S., Gogte P., Bahiram N., "Currency Recognition System Using Image Processing", International Research Journal of Engineering and Technology (IRJET), 4(3), 2599-2601, 2018.

[3] Ren Y., "Banknotes Recognition in real time using ANN", M.Sc. thesis presented to the university of Auckland, New Zeeland, 2017.

[4] Lee J., Hong H., Kim K., Park K., "A Survey on Banknote Recognition Methods by Various Sensors", Sensors Journal, 17(2), 235-359, 2017.

[5] Abu Doush L., AL-Btoush S., "Currency recognition using a smartphone: Comparison between color SIFT and gray scale SIFT algorithms", Journal of King Saud University- Computer and Information Sciences, 29(4), 484-492, 2017.

[6] Pham T., Park H., Kwon S., Park K., Jeong D., Yoon S., "Efficient Banknote Recognition Based on Selection of Discriminative Regions with One- 
Dimensional Visible-Light Line Sensor", Sensors, 16(3), 328-346, 2016.

[7] Bhurke C., Sirdeshmukh M., Kanitkar M.S., "Currency Recognition Using Image Processing", International Journal of Innovative Research in Computer and Communication Engineering, 3(5), 44184422, 2015.

[8] Saifullah S. M., Ananna A.R., Hossain S., Hossain J., Zishan S.R., "Currency Recognition System Using Image Processing", American Journal of Engineering Research, 4(11), 26-32, 2015.

[9] Sarfraz M., "An intelligent paper currency recognition system", Procedia Computer Science, 65(1), 538 - 545, 2015.

[10] Singh Reel P., Krishan G., Kotwal S., "Image Processing based Heuristic Analysis for Enhanced Currency Recognition", International Journal of Advancements in Technology, 2(1), 82-89, 2014.

[11] Shirsath A, Bharkad S, "Survey of Currency Recognition System Using Image Processing", International Journal of Computational Engineering Research, 3(7), 36-40, 2013.

[12] Jain V., Vijay R., "Indian Currency Denomination Identification Using Image Processing Technique", International Journal of Computer Science and Information Technologies, 4(1), 126 - 128, 2013.

[13] Mirza R., Nanda V., "Paper Currency Verification System Based on Characteristic Extraction Using Image Processing", International Journal of Engineering and Advanced Technology (IJEAT), 1(3), 68-71, 2012.

[14] Pathrabe T., Karmore S., "A Novel Approach of Embedded System for Indian Paper Currency Recognition", International Journal of Computer Trends and Technology- 4(8), 152-156, 2011.

[15] Wang Y, Ling s, "Currency recognition system using image processing", Bachelor Thesis, Akademin för teknik och miljö Högskolan i Gävle S-801 76 Gävle, Sweden, 2010.

[16] Debnath K., Ahmed S., Shahjahan M., "A Paper Currency Recognition System Using Negatively Correlated Neural
Network Ensemble", JOURNAL OF MULTIMEDIA, 5(6), 560-567, 2010.

[17] Hassanpour H., Farahabadi P., "Using Hidden Markov Models for paper currency recognition", Expert Systems with Applications, 36(6), 10105-10111, 2009.

[18] Liu X., Doermann D., "A Camera Phone Based Currency Reader for the Visually Impaired", ASSETS'08, ACM 978-159593-976-0/08/10, 2008.

[19] Zhang Er., Jiang Bo., Uan Jh., Bian Z, "Research on paper currency recognition by neural networks", Proceedings of the Second International Conference on Machine Learning and Cybernetics, Xian, 1(1), 2193-2197, 2003.

[20] Bay H., Tuytelaars T., Van Gool L., "SURF: Speeded Up Robust Features", In: Leonardis A., Bischof H., Pinz A. (eds) Computer Vision - ECCV 2006, Lecture Notes in Computer Science, 3951(1), 346359, 2006. 